\title{
Antineutrophil Cytoplasmic Antibody Frequency in Chronic Hepatitis B Patients
}

\author{
Turan Calhan, ${ }^{1}$ Abdurrahman Sahin, ${ }^{1}$ Resul Kahraman, ${ }^{1}$ Mustafa Erhan Altunoz, \\ Fatma Ozbakır, ${ }^{3}$ Kamil Ozdil, ${ }^{1}$ and Hacı Mehmet Sokmen ${ }^{1}$ \\ ${ }^{1}$ Department of Gastroenterology, Umraniye Education and Research Hospital, 34764 Istanbul, Turkey \\ ${ }^{2}$ Department of Gastroenterology, Haydarpasa Numune Education and Research Hospital, Istanbul, Turkey \\ ${ }^{3}$ Central Research Laboratory, Cerrahpasa Medical Faculty, University of Istanbul, Istanbul, Turkey \\ Correspondence should be addressed to Turan Calhan; trncalhan@hotmail.com
}

Received 24 June 2014; Revised 7 July 2014; Accepted 8 July 2014; Published 5 August 2014

Academic Editor: Giuseppe Murdaca

Copyright (C) 2014 Turan Calhan et al. This is an open access article distributed under the Creative Commons Attribution License, which permits unrestricted use, distribution, and reproduction in any medium, provided the original work is properly cited.

\begin{abstract}
Background. Chronic hepatitis $\mathrm{B}(\mathrm{CHB})$ is a viral disease, common across the world, and associated with several extraintestinal manifestations including vasculitis. Antineutrophil cytoplasmic antibodies (ANCAs) are sensitive and specific markers for vasculitides. There is limited data available in the literature on whether ANCA formation is stimulated by CHB infection. In the present study we aimed to identify the frequency of ANCA in CHB patients. Methods. A total of 174 subjects were included in the study (87 CHB patients, 87 control subjects). Perinuclear-ANCA (P-ANCA), cytoplasmic-ANCA (C-ANCA), myeloperoxidase ANCA (MPO-ANCA), and proteinase 3-ANCA (PR3-ANCA) were studied. IFA was used for P-ANCA and C-ANCA assays, and ELISA was used for MPO-ANCA and PR3-ANCA assays. Results. ANCA positivity was high in both groups ( $31 \%$ in the CHB group and $26 \%$ among controls). There were no significant differences between the groups for P-ANCA and MPO-ANCA $(P=0.6$ and $P=$ 0.6 , resp.). Frequency of borderline positive C-ANCA and all positive PR3-ANCA (positive + borderline positive) was significantly higher in the CHB group ( $P=0.009$ and $P=0.005$, resp.). Conclusions. In the present study, the frequency of ANCA was high in both groups. The CHB group had a relatively higher frequency of ANCA positivity compared to controls. Borderline positive C-ANCA and positive PR3-ANCA were significantly higher in the CHB group. These results suggest that ANCA may have a high prevalence in Turkey. Patients with CHB should be evaluated particularly for C-ANCA and PR3-ANCA in the presence of vasculitic complaints and lesions.
\end{abstract}

\section{Introduction}

Chronic hepatitis $\mathrm{B}(\mathrm{CHB})$ is a major cause of morbidity and mortality worldwide. Approximately 400 million individuals are infected with hepatitis B virus (HBV) globally $[1,2]$ and 1 million patients die annually due to HBV-associated complications, such as liver cirrhosis and hepatocellular carcinoma [3, 4]. CHB infection is associated with several extrahepatic manifestations, some of which are systemic (e.g., serum sickness and polyarteritis nodosa) and some are localized, such as dermatologic manifestations (e.g., urticaria, leukocytoclastic vasculitis, and essential mixed cryoglobulinemia) $[5,6]$. It has been demonstrated that antineutrophil cytoplasmic antibody (ANCA) positivity could be detected in many cases of vasculitis associated with viruses. These viruses are involved in the etiopathogenesis of ANCA positive vasculitis [7-15].

Antineutrophil cytoplasmic antibodies (ANCAs) are autoantibodies, usually of IgG structure, formed against azurophilic granules and lysosomal components in the cytoplasm of neutrophils and monocytes [16]. ANCAs are sensitive and specific markers of ANCA-related vasculitis and their frequency in the normal population is estimated to be low $(<5 \%)[17,18]$.

It is controversial whether vasculitis or other immune related presentations are related to ANCA in CHB patients. Moreover, it is important to determine whether HBV stimulates ANCA formation without triggering vasculitis or other immune related manifestations. There is insufficient data about this issue in CHB patients. We aimed in the 
present study to identify the frequency of perinuclear-ANCA (P-ANCA), cytoplasmic-ANCA (C-ANCA), myeloperoxidase-ANCA (MPO-ANCA), and proteinase 3-ANCA (PR3ANCA) subgroups of $\mathrm{CHB}$ patients in comparison with healthy controls.

\section{Materials and Methods}

This was a cross-sectional observational cohort study. From September 2011 to February 2012, 87 consecutive chronic hepatitis $B$ patients (male/female: $54 / 33$; mean age: $36 \pm 11$ years) who attended the hepatology outpatient clinic for routine followup were enrolled as the study population. 87 gender and age matched healthy individuals (male/female: 44/43; mean age: $37 \pm 11$ years) were enrolled as the control group. The inclusion criteria for the $\mathrm{CHB}$ group were patients with HBsAg positivity persisting for more than 6 months, patients above 16 years of age, patients without complications such as cirrhosis or hepatocellular carcinoma, patients without CHBrelated extrahepatic manifestations, patients with negative HCV and HIV tests, and patients without any malignancy and/or systemic disease (e.g., diabetes mellitus, hypertension, cerebrovascular disease, cancer, autoimmune disease, and rheumatic disease). Inclusion criteria for healthy controls were individuals above 16 years of age with no systemic disease who tested negative for complete viral hepatitis serology including anti-HBc IgG and hospitalized for other reasons, such as dyspeptic symptoms or volunteering hospital staff satisfying the same criteria. Individuals with high erythrocyte sedimentation rate and C-reactive protein measurement were excluded from either of the groups in the study. Demographic data, duration of disease, serum liver transaminase levels, HBV-DNA levels, HBeAg status, and antiviral treatment status for the $\mathrm{CHB}$ group were noted. Serum samples were obtained from all participants and kept frozen at $-80^{\circ} \mathrm{C}$ until the study date. Written informed consent was obtained from all patients. The study protocol was approved by the local ethics committee of our hospital (no. 14066, 23.09.2011).

P-ANCA and C-ANCA were analyzed with an indirect Immunofluorescence Assay (IFA) (ImmuGlo-IMMCO Diagnostic, Buffalo, NY, USA). MPO-ANCA and PR3-ANCA were analyzed with micro-ELISA (ImmuLisa-IMMCO Diagnostic, Buffalo, NY, USA). Results for IFA were categorized as negative, borderline positive, and positive. For ELISA, results less than $10 \mathrm{EU} / \mathrm{mL}$ were considered negative, results between 10 and $12.5 \mathrm{EU} / \mathrm{mL}$ were considered borderline positive, and results above $12.5 \mathrm{EU} / \mathrm{mL}$ were considered positive.

In this study, assuming that there would be a difference of $19 \%$ between the two groups for ANCA frequency, the sample size required to detect $80 \%$ power at $\alpha=0.05$ level required at least 80 subjects for each group. For statistical analyses, NCSS (Number Cruncher Statistical System) 2007 and PASS (Power Analysis and Sample Size) 2008 Statistical Software (Utah, USA) were used. Quantitative data with normal distribution was analyzed with Student's $t$-test and data without normal distribution was analyzed with MannWhitney $U$ test. Chi-square and Fisher's exact tests were used
TABLE 1: Comparison of the two groups by age, gender, and ANCA positivity (any ANCA positivity).

\begin{tabular}{lccc}
\hline & Control $(n=87)$ & CHB $(n=87)$ & $P$ \\
\hline Age, years (mean \pm SD) & $37.33 \pm 11.48$ & $36.99 \pm 11.27$ & 0.8 \\
\hline Gender & $n(\%)$ & $n(\%)$ & $P$ \\
$\quad$ Female & & & \\
$\quad$ Male & $43(49.4 \%)$ & $34(39.1 \%)$ & 0.1 \\
ANCA (any ANCA positivity) & $44(50.6 \%)$ & $53(60.9 \%)$ & \\
$\quad$ Positive & $23(26.4 \%)$ & $27(31 \%)$ & 0.5 \\
$\quad$ Negative & $64(73.6 \%)$ & $60(69 \%)$ & \\
\hline
\end{tabular}

CHB: chronic hepatitis B; ANCA: antineutrophil cytoplasmic antibody; and Mean \pm SD: mean \pm standard deviation.

to compare qualitative data. Statistical significance was set at $P<0.05$ with a $95 \%$ confidence interval.

\section{Results}

A total of 174 subjects were enrolled in the study. The study group and the control group did not differ significantly by age or gender. Comparison of the two groups for ANCA positivity (total ANCA positivity) did not yield any significant differences. Age, gender, and ANCA positivity information of the groups are provided in Table 1.

Comparisons of the two groups for positive, borderline positive, and any (all) positive (positive + borderline positive cases) results for P-ANCA and MPO-ANCA did not reveal significant differences. Frequency of borderline positive CANCA and of any (all) positive PR3-ANCA (positive + borderline positive cases) was significantly higher in the $\mathrm{CHB}$ group ( $P=0.009$ and $P=0.005$, resp.). Detailed comparative ANCA data of the two groups are given in Table 2.

In the $\mathrm{CHB}$ group, the mean disease duration was $7 \pm 4$ years, mean aspartate transaminase (AST) level was $30 \pm$ $20 \mathrm{I} / \mathrm{U}$, mean alanine transaminase (ALT) level was $45 \pm$ $47 \mathrm{I} / \mathrm{U}, 84 \%(73 / 87)$ of the patients in this group were HBeAg negative, and 56\% (49/87) had HBV-DNA levels of $\leq 2000 \mathrm{IU} / \mathrm{mL}$. Among these, 39\% (34/87) were receiving antiviral therapy (12 with entecavir, 19 with tenofovir, and 3 with lamivudine), while $61 \%$ (53/87) were not receiving antiviral treatment. ANCA positive cases in the CHB group were compared for disease duration, $\mathrm{HBeAg}$ positivity, antiviral therapy, and HBV-DNA levels, yielding no significant relationship.

\section{Discussion}

The role of hepatotropic viruses such as HBV and hepatitis $\mathrm{C}$ virus (HCV) with similar immunopathogenesis in ANCA development and ANCA-related vasculitis etiology is a current topic of investigation. Available data on this topic is insufficient and further research is necessary. In a study of chronic viral hepatitis patients, the frequency of ANCA was higher in an HCV group compared to controls although the same was not observed for the HBV group 
TABLE 2: Comparison of the two groups by P-ANCA, C-ANCA, MPO-ANCA, and PR3-ANCA.

\begin{tabular}{lccc}
\hline & Control $(n, \%)$ & CHB $(n, \%)$ & $P$ \\
\hline P-ANCA & & & \\
Negative & $80(92 \%)$ & $78(89.7 \%)$ & - \\
Positive & $6(6.9 \%)$ & $3(3.4 \%)$ & 0.4 \\
Borderline positive & $1(1.1 \%)$ & $6(6.9 \%)$ & 0.1 \\
All positive (any positivity) & $7(8 \%)$ & $9(10.3 \%)$ & 0.6 \\
C-ANCA & & & \\
Negative & $78(89.7 \%)$ & $71(81.6 \%)$ & - \\
Positive & $7(8 \%)$ & $5(5.7 \%)$ & 0.5 \\
Borderline positive & $2(2.3 \%)$ & $11(12.6 \%)$ & $\mathbf{0 . 0 0 9}$ \\
All positive (any positivity) & $9(10.3 \%)$ & $16(18.4 \%)$ & 0.1 \\
MPO-ANCA & & & \\
Negative & $78(89.7 \%)$ & $80(92 \%)$ & - \\
Positive & $5(5.7 \%)$ & $2(2.3 \%)$ & 0.4 \\
Borderline positive & $4(4.6 \%)$ & $5(5.7 \%)$ & 1 \\
All positive (any positivity) & $9(10.3 \%)$ & $7(8 \%)$ & 0.6 \\
PR3-ANCA & & & \\
Negative & $81(93.1 \%)$ & $68(78.2 \%)$ & - \\
Positive & $1(1.1 \%)$ & $7(8 \%)$ & 0.06 \\
Borderline positive & $5(5.7 \%)$ & $12(13.8 \%)$ & 0.07 \\
All positive (any positivity) & $6(6.9 \%)$ & $19(21.8 \%)$ & $\mathbf{0 . 0 0 5}$ \\
\hline
\end{tabular}

CHB: chronic hepatitis B; ANCA: antineutrophil cytoplasmic antibody; P: perinuclear; C: cytoplasmic; MPO: myeloperoxidase; and PR3: proteinase 3.

[19]. A study conducted on HCV patients suggests that PR3ANCA positivity was more common and that HCV could be a cause for ANCA-related vasculitis [20]. In two case reports of Wegener granulomatosis patients with hepatitis C, one was positive for MPO-ANCA [21], while another patient had a high titer of PR3-ANCA positivity [22]. In both reports the role of HCV in systemic vasculitis was underlined. Another case presentation discussed concurrent Churg-Strauss syndrome and HBV in an ANCA positive patient [11].

In this study, $31 \%$ of the patients (27/87) in the CHB group had ANCA positivity (any ANCA positivity). These results demonstrate a high prevalence of ANCA positivity in the $\mathrm{CHB}$ group. The most significant difference between $\mathrm{CHB}$ and control groups arises from borderline positivity for $\mathrm{C}$ ANCA and PR3-ANCA (for C-ANCA: 11 with borderline positive versus 2 in controls; for PR3-ANCA: 12 with borderline positive versus 5 in controls). The borderline positivity in the CHB group might be the result of the patient selection criteria. The patients with rheumatic manifestations and elevated inflammatory markers were excluded. Therefore the remaining patients were the ones with borderline positivity with subclinical manifestations. In other words, the higher number of borderline positive subjects in our study could suggest that $\mathrm{CHB}$ stimulates ANCA development without exhibiting clinical manifestations.

ANCA is divided into three patterns by indirect immunofluorescence: C-ANCA, P-ANCA, and atypical ANCA. Classical P-ANCA occurs with antibodies directed to MPO. P-ANCA without nuclear extension occurs with antibodies to bacterial permeability increasing factor, cathepsin G, elastase, lactoferrin, and lysozyme. The most common antigens used on an ELISA are MPO and PR3, so we investigated MPO and PR3 subgroups in the present study [23]. We found a higher positivity frequency only for both C-ANCA and PR3-ANCA patterns in CHB patients. Taking into account our results, it is suggested that $\mathrm{CHB}$ may cause development of antigens that influence cytoplasmic ANCA patterns.

The pathogenesis of virus-related vasculitides is thought to be heterogeneous and at least two mechanisms have been suggested to be involved; in the first mechanism, viral replication induces direct damage to the blood vessel wall leading to viral arteritis, and the second is a theory that suggests that vascular damage could be associated with the immune mechanism [24]. Immunologic mechanisms that are involved in the pathogenesis may be related to cellular and/or humoral immunity and may be in the form of immunocomplex deposition $[25,26]$. Extrahepatic manifestations seen in HBV are believed to result mostly from immunological pathways $[6,27]$. It has been suggested that persistent antigen load secondary to chronic infection and high viral replication rate lead to excessive formation of immunocomplexes, and these circulating immunocomplexes deposited in intermediate and small-sized vessels initiate vasculitic processes $[28,29]$. Vasculitic lesion development in $\mathrm{HBV}$ is believed to be associated rather with the $\mathrm{HBeAg}$ antigen or viral replication [30]. In our study, $84 \%$ of the patients were HBeAg negative and 56\% had HBV-DNA levels below $2000 \mathrm{IU} / \mathrm{mL}$. These findings may account for the lack of significance for high total ANCA, despite the significant rates of positivity identified for C-ANCA and PR3-ANCA in the $\mathrm{CHB}$ group. For a better understanding of the ANCA and HBV relationship, studies involving patient groups with high viral load and who are HBeAg $(+)$ could provide valuable information.

Adequate data on $\mathrm{CHB}$ and the incidence of ANCA in healthy individuals in Turkey is not available. The high ANCA prevalence in the healthy control group (26\%) was a remarkable finding of our study. In both groups, positive samples for ANCA were studied at least two times. Samples were then studied for antinuclear antibody (ANA). After the proven negative results for ANA, the sample was considered ANCA positive. This ANCA prevalence of $26 \%$ in our controls versus the $<5 \%$ value reported in the literature makes it difficult to determine whether ANCA positivity in $\mathrm{CHB}$ patients is due to CHB itself or a higher prevalence of ANCA in the Turkish population. This complicates the assessment of the effect of HBV on ANCA despite the significantly higher rates of PR3-ANCA and C-ANCA.

There are some limitations of this study. Firstly, we did not evaluate atypical ANCA patterns because these patterns constitute approximately $5 \%$ of all ANCA, and the most common antigens are PR3 and MPO. Another limitation is that this study was conducted on $\mathrm{CHB}$ patients for which more than half were $\mathrm{HBe}$ antigen negative. Further studies on HBe antigen positive patient groups are needed. This study is a single center study that was conducted with a small number of patients. Thus there is also a need for a larger series. 


\section{Conclusions}

ANCA frequency was high in both groups in our study. The $\mathrm{CHB}$ group had a relatively higher number of ANCA positive individuals compared to the control group. Borderline positive C-ANCA and positive PR3-ANCA (positive + borderline positive cases) were significantly higher in the $\mathrm{CHB}$ group. These results indicate that patients with $\mathrm{CHB}$ infection should be evaluated particularly for C-ANCA and PR3-ANCA in the presence of vasculitic complaints and lesions. However, larger studies with a larger number of subjects are needed to verify these data.

\section{Conflict of Interests}

The authors declare that there is no conflict of interests regarding the publication of this paper.

\section{Authors' Contribution}

Turan Calhan designed the study and wrote the paper; Abdurrahman Sahin, Resul Kahraman, and Mustafa Erhan Altunoz helped in collecting the data of subjects; Fatma Ozbakır assessed the biochemical analysis; Kamil Ozdil and Hac1 Mehmet Sokmen participated in coordination and drafted the paper. All authors read and approved the final paper.

\section{References}

[1] W. M. Lee, "Hepatitis B virus infection," The New England Journal of Medicine, vol. 337, no. 24, pp. 1733-1745, 1997.

[2] C. L. Lai, V. Ratziu, M. Yuen, and T. Poynard, "Viral hepatitis B," The Lancet, vol. 362, no. 9401, pp. 2089-2094, 2003.

[3] D. Lavanchy, "Hepatitis B virus epidemiology, disease burden, treatment, arid current and emerging prevention and control measures," Journal of Viral Hepatitis, vol. 11, no. 2, pp. 97-107, 2004.

[4] M. J. Alter, S. C. Hadler, H. S. Margolis et al., "The changing epidemiology of hepatitis B in the United States. Need for alternative vaccination strategies," The Journal of the American Medical Association, vol. 263, no. 9, pp. 1218-1222, 1990.

[5] R. A. Willson, "Extrahepatic manifestations of chronic viral hepatitis," The American Journal of Gastroenterology, vol. 92, no. 1, pp. 3-17, 1997.

[6] P. Cacoub and B. Terrier, "Hepatitis B-Related Autoimmune Manifestations," Rheumatic Disease Clinics of North America, vol. 35, no. 1, pp. 125-137, 2009.

[7] T. N. Chou, T. C. Hsu, R. M. Chen, L. I. Lin, and G. J. Tsay, "Parvovirus B19 infection associated with the production of antineutrophil cytoplasmic antibody (ANCA) and anticardiolipin antibody (aCL)," Lupus, vol. 9, no. 7, pp. 551-554, 2000.

[8] M. Papi, B. Didona, O. de Pità, M. Gantcheva, and L. M. Chinni, "Chronic hepatitis $\mathrm{C}$ virus infection, mixed cryoglobulinaemia, leukocytoclastic vasculitis and antineutrophil cytoplasmic antibodies," Lupus, vol. 6, no. 9, pp. 737-738, 1997.

[9] J. A. Savige, L. Chang, and S. M. Crowe, "Anti-neutrophil cytoplasm antibodies in HIV infection," Advances in Experimental Medicine and Biology, vol. 336, pp. 349-352, 1993.
[10] C. G. M. Kallenberg and H. Tadema, "Vasculitis and infections: Contribution to the issue of autoimmunity reviews devoted to "autoimmunity and infection"', Autoimmunity Reviews, vol. 8, no. 1, pp. 29-32, 2008.

[11] D. S. Domiciano, S. K. Shinjo, and M. Levy-Neto, "Churgstrauss syndrome and active chronic hepatitis B virus infection: coincidence or association?" Clinics, vol. 65, no. 3, pp. 335-336, 2010.

[12] L. M. Cooper and J. A. K. Patterson, "Allergic granulomatosis and angiitis of Churg-Strauss. Case report in a patient with antibodies to human immunodeficiency virus and hepatitis B virus," International Journal of Dermatology, vol. 28, no. 9, pp. 597-599, 1989.

[13] B. Bonaci-Nikolic, S. Andrejevic, M. Pavlovic, Z. Dimcic, B. Ivanovic, and M. Nikolic, "Prolonged infections associated with antineutrophil cytoplasmic antibodies specific to proteinase 3 and myeloperoxidase: diagnostic and therapeutic challenge," Clinical Rheumatology, vol. 29, no. 8, pp. 893-904, 2010.

[14] M. Cojocaru, I. M. Cojocaru, and S. A. Iacob, "Prevalence of anti-neutrophil cytoplasmic antibodies in patients with chronic hepatitis $\mathrm{C}$ infection associated mixed cryoglobulinemia," Romanian Journal of Internal Medicine, vol. 44, no. 4, pp. 427-431, 2006.

[15] Y. Bayraktar, M. Bayraktar, A. Gurakar, T. I. Hassanein, and D. H. Van Thiel, "A comparison of the prevalence of autoantibodies in individuals with chronic hepatitis $\mathrm{C}$ and those with autoimmune hepatitis: the role of interferon in the development of autoimmune diseases," Hepato-Gastroenterology, vol. 44, no. 14, pp. 417-425, 1997.

[16] A. Wiik, "Delineation of a standard procedure for indirect immunofluorescence detection of ANCA," APMIS Supplement, vol. 6, pp. 12-13, 1989.

[17] P. A. Merkel, R. P. Polisson, Y. Chang, S. J. Skates, and J. L. Niles, "Prevalence of antineutrophil cytoplasmic antibodies in a large inception cohort of patients with connective tissue disease," Annals of Internal Medicine, vol. 126, no. 11, pp. 866-873, 1997.

[18] J. F. Maillefert, P. Pfitzenmeyer, M. Thenet et al., "Prevalence of ANCA in a hospitalized elderly French population," Clinical and Experimental Rheumatology, vol. 15, no. 6, pp. 603-607, 1997.

[19] M. Ghonaim, A. Al-Ghamdi, H. El-Bana et al., "Autoantibodies in chronic liver disease," The Egyptian Journal of Immunology, vol. 12, no. 2, pp. 101-111, 2005.

[20] Y.-Y. Wu, T.-C. Hsu, T.-Y. Chen et al., "Proteinase 3 and dihydrolipoamide dehydrogenase (E3) are major autoantigens in hepatitis $\mathrm{C}$ virus (HCV) infection," Clinical and Experimental Immunology, vol. 128, no. 2, pp. 347-352, 2002.

[21] M. Yasuda, H. Araki, Y. Fujitomo et al., "A case of MPOANCA-positive Wegener's granulomatosis with hepatitis C virus infection," Japanese Journal of Nephrology, vol. 53, no. 7, pp. 1053-1058, 2011.

[22] J. Marx, V. Schwenger, N. Blank, W. Stremmel, and J. Encke, "Hemoptysis and acute renal failure in a 29 -year-old patient with chronic hepatitis C infection," Internist, vol. 49, no. 9, pp. 1120-1125, 2008.

[23] J. Savige, D. Davies, R. J. Falk, J. C. Jennette, and A. Wiik, "Antineutrophil cytoplasmic antibodies and associated diseases: a review of the clinical and laboratory features," Kidney International, vol. 57, no. 3, pp. 846-862, 2000.

[24] T. Michalak, "Immune complexes of hepatitis B surface antigen in the pathogenesis of periarteritis nodosa. A study of seven necropsy cases," The American Journal of Pathology, vol. 90, no. 3, pp. 619-632, 1978. 
[25] A. M. Prince and C. Trepo, "Role of immune complexes involving $\mathrm{SH}$ antigen in pathogenesis of chronic active hepatitis and polyarteritis nodosa," The Lancet, vol. 1, no. 7713, pp. 1309$1312,1971$.

[26] L. H. Calabrese, "The rheumatic manifestations of infection with the human immunodeficiency virus," Seminars in Arthritis and Rheumatism, vol. 18, no. 4, pp. 225-239, 1989.

[27] D. J. Gocke, K. Hsu, C. Morgan, S. Bombardieri, M. Lockshin, and C. L. Christian, "Association between polyarteritis and Australia antigen," The Lancet, vol. 2, no. 7684, pp. 1149-1153, 1970.

[28] K. H. Fye, M. J. Becker, A. N. Theofilopoulos, H. Moutsopoulos, J. L. Feldman, and N. Talal, "Immune complexes in hepatitis B antigen associated periarteritis nodosa. Detection by antibody dependent cell mediated cytotoxicity and the Raji cell assay," The American Journal of Medicine, vol. 62, no. 5, pp. 783-791, 1977.

[29] A. Mason, J. Theal, V. Bain, E. Adams, and R. Perrillo, "Hepatitis $B$ virus replication in damaged endothelial tissues of patients with extrahepatic disease," The American Journal of Gastroenterology, vol. 100, no. 4, pp. 972-976, 2005.

[30] A. Mason, "Role of viral replication in extrahepatic syndromes related to hepatitis B virus infection," Minerva Gastroenterologica e Dietologica, vol. 52, no. 1, pp. 53-66, 2006. 


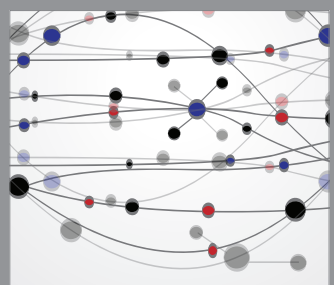

The Scientific World Journal
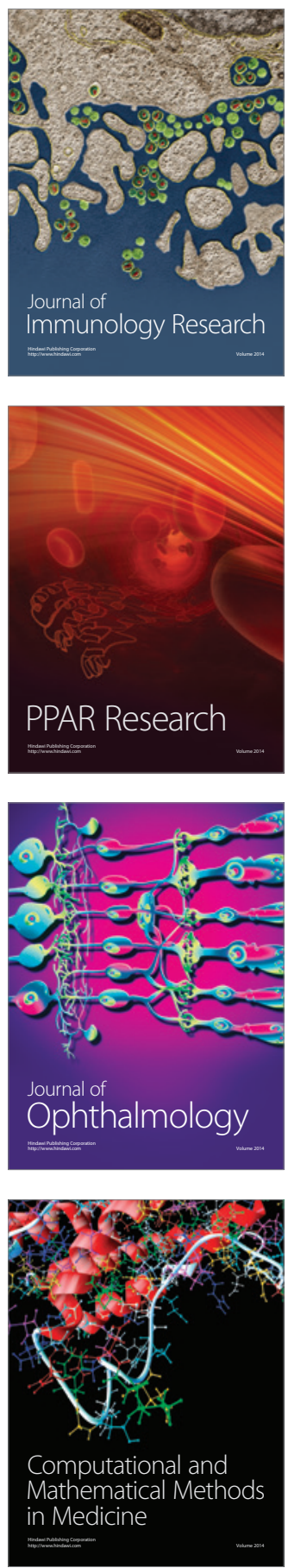

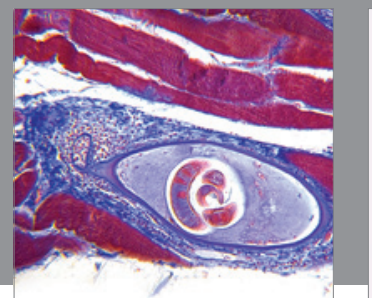

Gastroenterology

Research and Practice
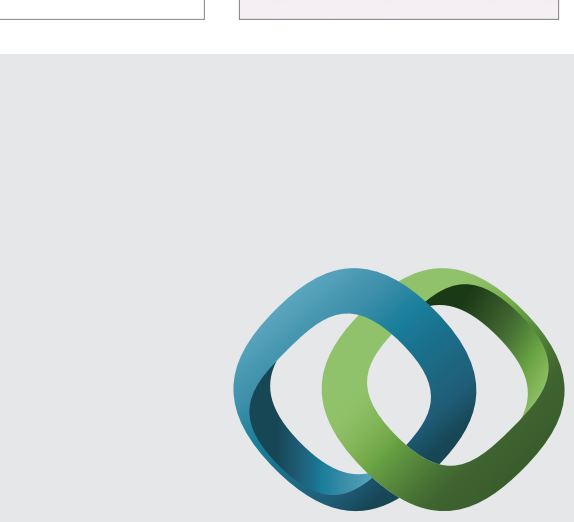

\section{Hindawi}

Submit your manuscripts at

http://www.hindawi.com
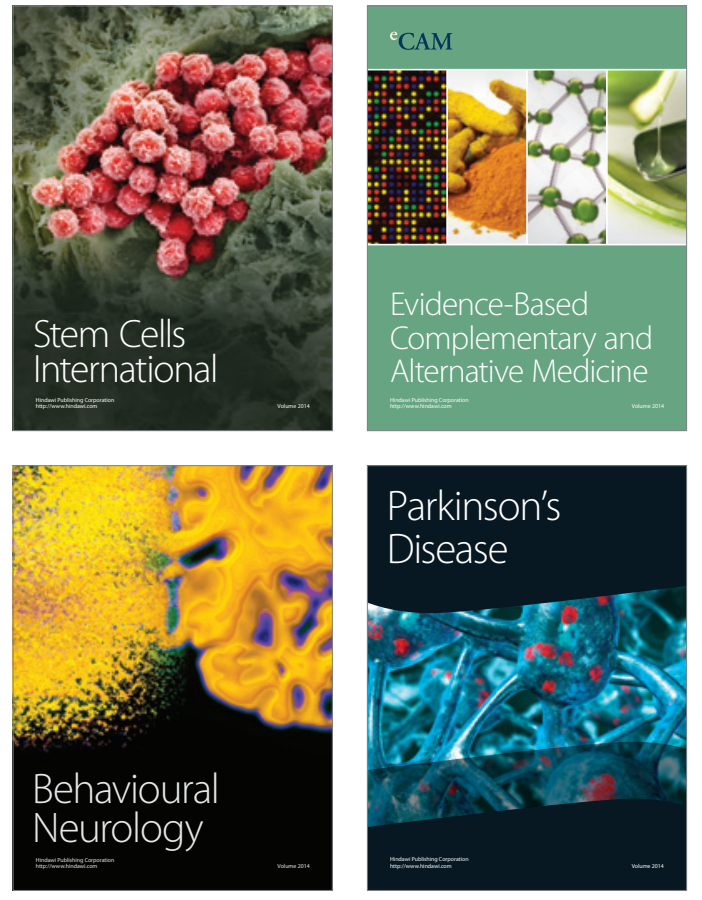
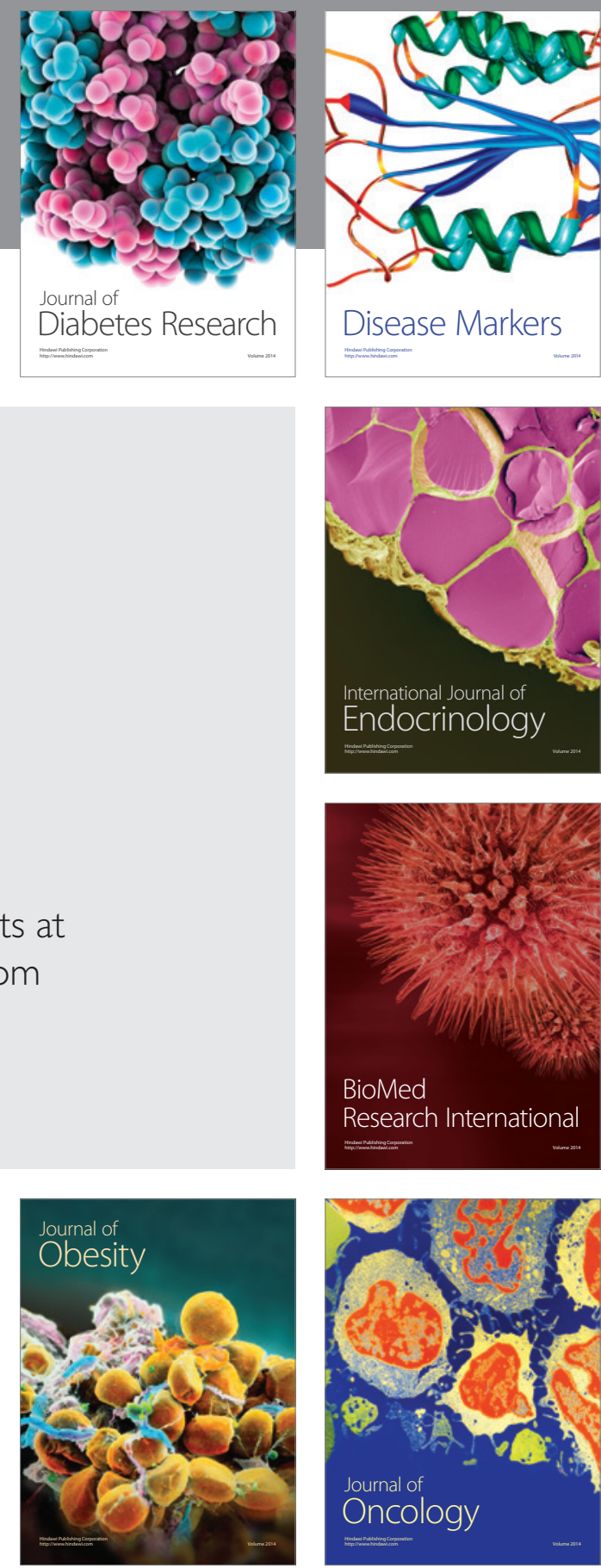

Disease Markers
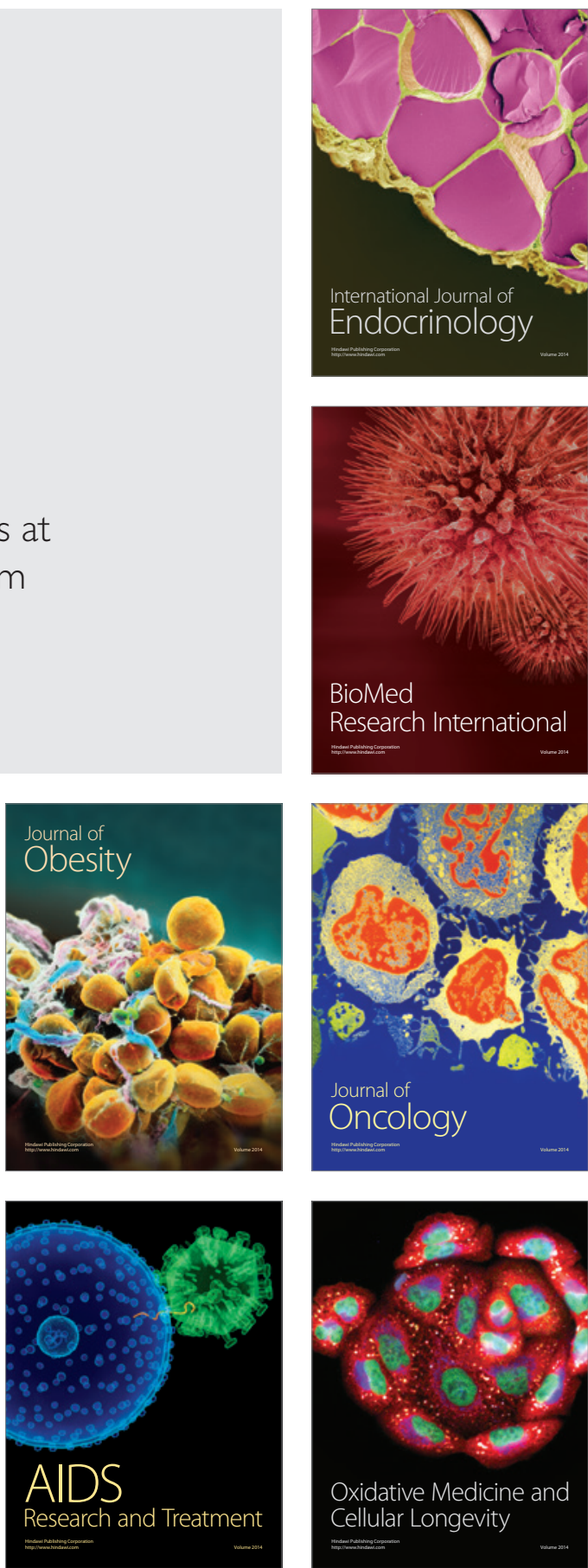